\title{
Nanobelt Self-assembly from an Organic $n$-Type Semiconductor: Propoxyethyl-PTCDI
}

\author{
Kaushik Balakrishnan ${ }^{1}$, Aniket Datar ${ }^{1}$, Randy Oitker ${ }^{1}$, Hao Chen $^{2}$, Jianmin Zuo ${ }^{2}$, Ling Zang*, \\ ${ }^{1}$ Department of Chemistry and Biochemistry, Southern Illinois University, \\ Carbondale, IL 62901, and \\ ${ }^{2}$ Department of Materials Science and Engineering and Materials Research Laboratory, University \\ of Illinois at Urbana-Champaign, Urbana, Illinois 61801
}

\section{Supporting Information}

\section{Materials}

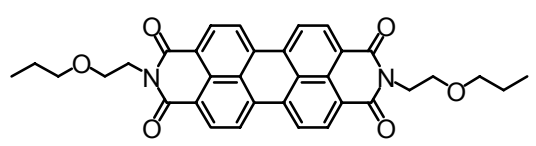

N,N'-di(propoxyethyl)-perylene-3,4,9,10-tetracarboxylic diimide (PE-PTCDI)

This compound was synthesized following the standard condensation method developed by Langhals. ${ }^{1}$ Briefly, $0.62 \mathrm{~g}(1.56 \mathrm{mmol})$ perylene-3,4,9,10-tetracarboxylic dianhydride (Aldrich), 1.6 $\mathrm{g}(15.5 \mathrm{mmol})$ propoxyethylamine (TCI), and $5 \mathrm{~g}$ imidazole were heated under argon at $100{ }^{\circ} \mathrm{C}$ for $4 \mathrm{~h}$. The reaction mixture was cooled to room temperature and dispersed in $100 \mathrm{~mL}$ ethanol followed by addition of $300 \mathrm{~mL} 2 \mathrm{M} \mathrm{HCl}$. The mixture was stirred overnight. The resulting red solid was collected by vacuum filtration through a $0.45 \mu \mathrm{m}$ membrane filter (Osmonics). It was washed thoroughly with distilled water until the $\mathrm{pH}$ of washings turned to be neutral. The collected solid was dried in vacuum at $60{ }^{\circ} \mathrm{C}$. TLC: $\mathrm{R}_{\mathrm{f}}\left(\right.$ silica gel $/ \mathrm{CHCl}_{3}: \mathrm{n}$-butanol 95:5) $=0.36$.

${ }^{1} \mathrm{H}-\mathrm{NMR}\left(\mathrm{CDCl}_{3}\right): \delta=0.94\left(\mathrm{t}, 6 \mathrm{H}, 2 \mathrm{CH}_{3}\right), 1.56\left(\mathrm{~m}, 4 \mathrm{H}, 2 \mathrm{CH}_{2}-\mathrm{CH}_{3}\right), 3.49\left(\mathrm{t}, 4 \mathrm{H}, 2 \mathrm{O}-\mathrm{CH}_{2}\right), 3.8(\mathrm{t}$, $\left.4 \mathrm{H}, 2 \mathrm{CH}_{2}-\mathrm{O}\right), 4.48\left(\mathrm{t}, 4 \mathrm{H}, 2 \mathrm{~N}-\mathrm{CH}_{2}\right), 8.68(2 \mathrm{~d}, 8 \mathrm{H}$, perylene).

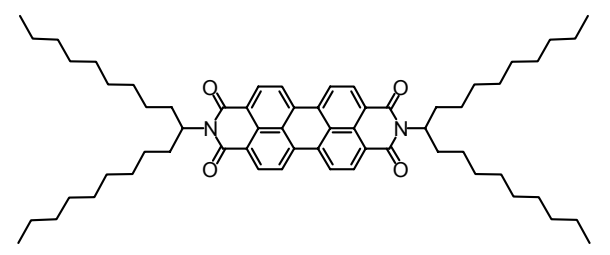

N,N'-di(1-nonyldecyl)perylene-3,4,9,10-tetracarboxylic diimide (ND-PTCDI)

This compound was synthesized following the standard condensation method developed by Langhals. ${ }^{1}$ Briefly, $1.44 \mathrm{~g}(3.7 \mathrm{mmol})$ perylene-3,4,9,10-tetracarboxylic dianhydride (Aldrich), 2.67 g (9.4 mmol) 1-nonyldecylamine (synthesized from di- $n$-nonyl ketone and ammonium formate according to literature, Chem. Abstr. 1965, 63, 13112f ), and $6 \mathrm{~g}$ imidazole were heated under argon at $120^{\circ} \mathrm{C}$ for $3 \mathrm{~h}$. The reaction mixture was cooled to room temperature and dispersed in $100 \mathrm{~mL}$ ethanol followed by addition of $300 \mathrm{~mL} 2 \mathrm{M} \mathrm{HCl}$. The mixture was stirred overnight. The resulting 
red solid was collected by vacuum filtration through a $0.45 \mu \mathrm{m}$ membrane filter (Osmonics). It was washed thoroughly with distilled water until the $\mathrm{pH}$ of washings turned to be neutral. The collected solid was dried in vacuum at $100{ }^{\circ} \mathrm{C}$. $\mathrm{R}_{\mathrm{f}}\left(\right.$ silica $\left.\mathrm{gel} / \mathrm{CHCl}_{3}\right)=0.9$.

${ }^{1} \mathrm{H}-\mathrm{NMR}\left(\mathrm{CDCl}_{3}\right): \delta=0.83\left(\mathrm{t}, 12 \mathrm{H}, 4 \mathrm{CH}_{3}\right), 1.25\left(\mathrm{~m}, 56 \mathrm{H}, 28 \mathrm{CH}_{2}\right), 1.86\left(\mathrm{~m}, 4 \mathrm{H}, 2 \alpha-\mathrm{CH}_{2}\right), 2.24(\mathrm{~m}$, $\left.4 \mathrm{H}, 2 \alpha-\mathrm{CH}_{2}\right), 5.18(\mathrm{~m}, 2 \mathrm{H}, 2 \mathrm{CH}), 8.67$ (m, 8H, perylene).

\section{Self-assembly Processing}

Self-assembly of the nanobelts of propoxyethyl-PTCDI was performed through a solvent exchange processing, which transfers the molecule from a "good" solvent (such as chloroform) into a "bad" solvent (such as methanol) where the molecule has limited solubility and thus self-assembly occurs via molecular stacking. Such a self-assembly approach takes the advantage of the strong intermolecular $\pi-\pi$ interaction, which is enhanced in a solvent where the molecule has minimum interaction with the solvent. Similar methods have previously been used for self-assembling onedimensional nanostructures of macro-aromatic molecules. ${ }^{2-8}$

In this study, we used a solution injection method to conduct the self-assembly in methanol. Typically, a minimum volume $(5-125 \mu \mathrm{L})$ of concentrated chloroform solution $(1 \mathrm{mM})$ of propoxyethyl-PTCDI was injected rapidly into a larger volume $(5 \mathrm{~mL})$ of methanol, followed by immediate mixing with pipette. Thus, the final concentration of the molecule was $5-25 \mu \mathrm{M}$, and the mixed solution contains only slight amount of chloroform $(<2.5 \%)$. Effective self-assembly of nanobelts should be conducted in methanol with minimum amount of chloroform $(<5 \%)$. Such a solvent provides limited solubility for propoxyethyl-PTCDI, but on the other hand leads to favorable molecular stacking. If the volume ratio of chloroform is above $10 \%$, there will be significant amount of free molecules existing in the nanobelt suspension. When the ratio of chloroform is above $50 \%$, the binary solvent becomes a good solvent for propoxyethyl-PTCDI, and there is no formation of crystal phase (i.e. no self-assembly). For example, there was no change observed for the UV-vis absorption spectra of a $5 \mu \mathrm{M}$ solution in $1 / 1 \mathrm{chloroform} / \mathrm{methanol}$. This is in contrast to what we observed in methanol (Fig. 1). All preparations were carried out under ambient conditions, as were the spectral and microscopy measurements (described below).
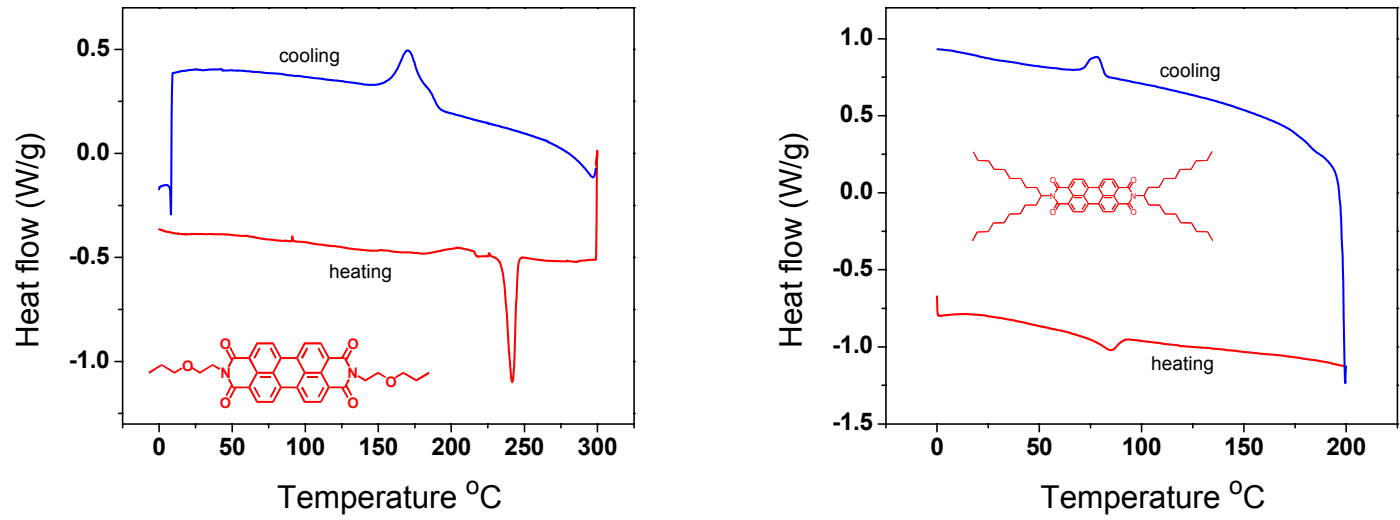

Figure S1. Differential scanning calorimetry (DSC) traces of the two molecules, PE-PTCDI and ND-PTCDI, which show different phase transition temperatures (melting point) due to the different molecular packing conformation. Both heating and cooling curves are depicted.

Methanol seems to be an ideal solvent for 1-D self-assembly of propoxyethyl-PTCDI. On one hand, methanol provides little solubility for the molecule due to $\pi-\pi$ stacking; on the other hand, 
there is sufficient side-chain interaction (hydrogen bonding) between methanol and the molecule. When dispersed in methanol, propoxyethyl-PTCDI prefers 1-D packing through $\pi-\pi$ interaction, leading to maximum exposure of side chains to solvent phase. The slow process of self-packing (Fig. 1) leads to optimal Oswald ripening of the nanobelts, and thus produces uniform nanobelt structures. Direct drying from a chloroform solution resulted in dark red solid of propoxyethylPTCDI with irregular shapes of big powders or chunks. This is consistent with our recent AFM measurement of the propoxyethyl-PTCDI sample made by drop-casting a concentrate chloroform solution $(25$ and $100 \mu \mathrm{M})$ on mica, for which only irregular aggregates were observed.

\section{DSC Measurement}

Differential Scanning Calorimetry (DSC) was performed using a Mettler-Toledo DSC 821. The sample was heated \& cooled for two cycles between zero and a certain temperature above the melting point. DSC traces collected from the second cycle were presented in Fig. S1. For NDPTCDI, the heating and cooling curves show a consistent transition temperature, $84{ }^{\circ} \mathrm{C}$, which is the melting point as measured elsewhere. For PE-PTCDI, the heating curve shows a single phase transition temperature $\left(242{ }^{\circ} \mathrm{C}\right)$ corresponding to the melting point. During the cooling, the phase transition shifted to a lower temperature and a small shoulder appeared just before the major peak. This is reminiscent of the properties of liquid crystal materials. ${ }^{9,10}$ However, due to the superior $\pi-\pi$ stacking over the side chain interdigitation, the liquid crystal phase is not prominent for PE-PTCDI materials.

\section{Spectral Characterization}

Due to the steric hindrance of the swallow-tail-like side chains, ND-PTCDI shows the highest solubility (compared to other PTCDI molecules) and strong fluorescence $(\phi=100 \%)$ in most organic solvents. It is thus often used as a standard for measuring the fluorescence quantum yield of new molecules. Here we used it as a reference molecule for PE-PTCDI to evaluate the side chain effect on the dimensional structure of molecular packing (self-assembly). Because of the different steric hindrance of the side chain, the two molecules showed completely different dimensional structures of self-assembly.
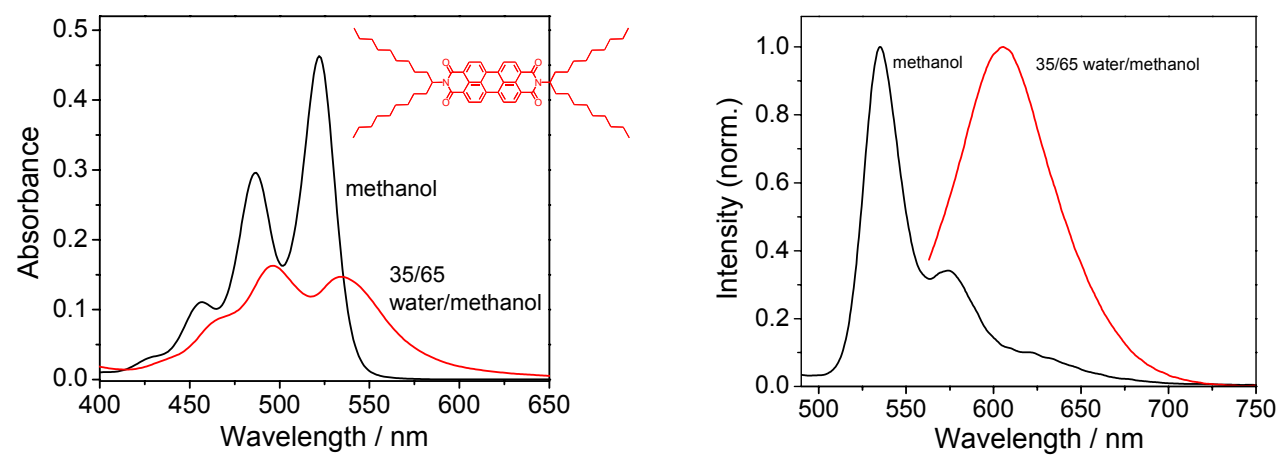

Figure S2. Absorption (left) and fluorescence (right) spectra of ND-PTCDI in pure methanol and water/methanol (35/65) binary solvent. In methanol, the fluorescence was recorded with excitation at $450 \mathrm{~nm}$. In the binary solvent, the excitation wavelength was selected at $550 \mathrm{~nm}$, where most absorption is due to the crystal phase.

When dispersed in a water/methanol (35/65) binary solvent, ND-PTCDI molecules turn to crystallize. This is evidenced by the strong absorption at longer wavelength and the enhanced 0-1 
transition at $495 \mathrm{~nm}$ (Fig. S2). Excitation at the crystal phase $(550 \mathrm{~nm})$ results in bright red fluorescence centered at $605 \mathrm{~nm}$. This is in contrast to the case of PE-PTCDI, where no fluorescence was detected from the crystal phase. The different observations imply different packing conformation for the two molecules. As discussed in the Communication, the short side chain of PE-PTCDI favors strong $\pi-\pi$ interaction and thus optimal face-to-face molecular stacking, which leads to a forbidden low energy excitonic transition. When the molecular packing is distorted or weakened by the side chain modulation, like the case of ND-PTCDI, the low energy excitonic transition will become allowable as observed in Fig. S2.

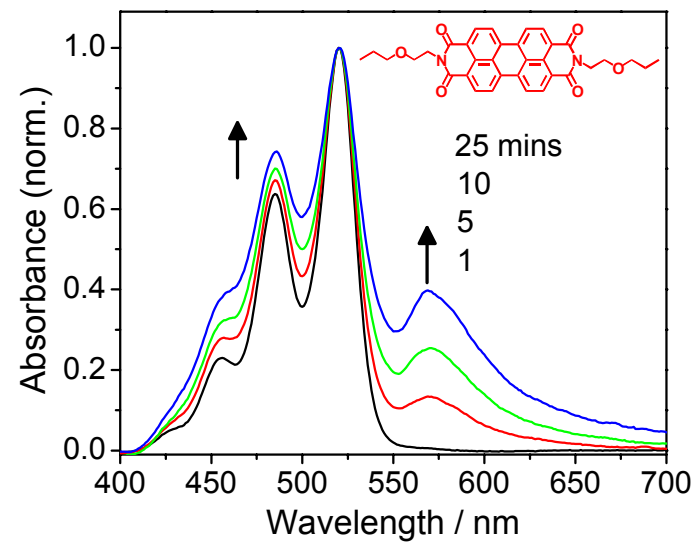

Figure S3. Normalized data of Fig. 1 in the Communication.

Fig. S3 shows the normalized absorption spectra of PE-PTCDI in methanol recorded at different time intervals following injection of the chloroform solution. The enhanced $0-1,0-2$ and $0-3$ transitions at 485, 455 and 426, respectively, are clearly revealed.

\section{SEM Measurement}

SEM measurement was performed with a Hitachi S570 (operated at $10 \mathrm{kV}$ ). The sample was prepared by casting a methanol solution of PE-PTCDI nanobelts $(25 \mu \mathrm{M}$, aged 1 hour $)$ onto a clean glass slide, followed by drying in air. The dried sample was then annealed overnight in an oven at $45^{\circ} \mathrm{C}$, followed by coating of gold.
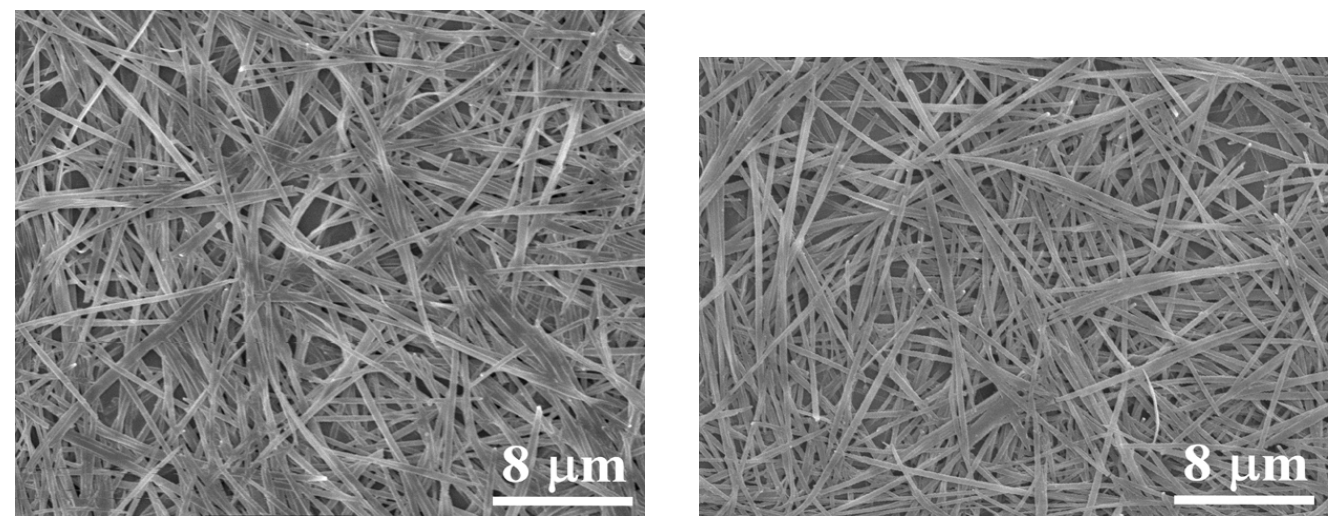

Figure S4. Large area SEM image of nanobelt piles.

Large area nanobelt piles were found over the surface, and they showed quite narrow size (diameter) distribution. Most of the nanobelts were longer than $10 \mu \mathrm{m}$ (Figure S4). The cross 
section of the nanobelt is of rectangular shape with a width-to-height ratio of approximate $4: 1$. This can be seen from a broken nanobelt which is wrapped onto a crystal chunk (Figure S5). The 4:1 rectangular cross section can also be revealed by AFM imaging as discussed below. In some areas, some nanobelts show twisted conformation with the edge lifted up perpendicular to the substrate (Figure S6). This proves the belt like shape of the nanomotifs.

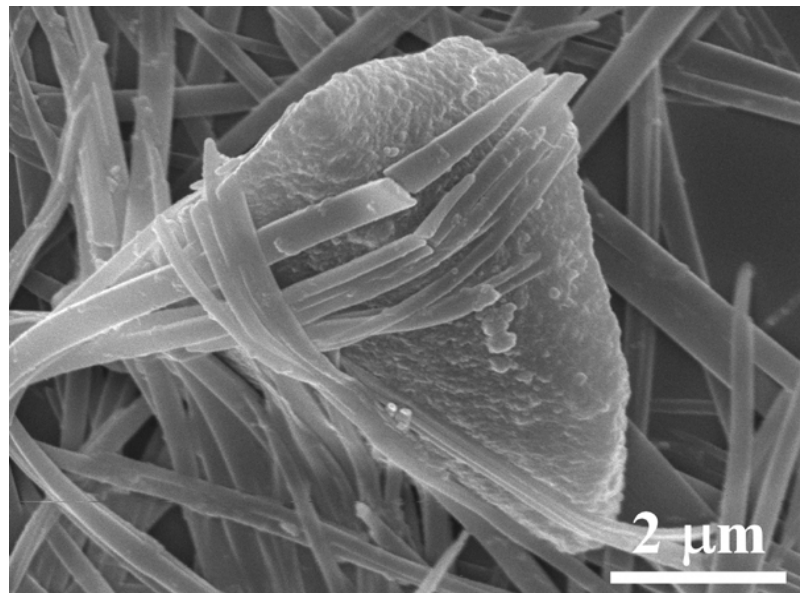

Figure S5. SEM image showing a broken nanobelt wrapped on a crystal chunk. The approximately rectangular shape of cross section can be seen.

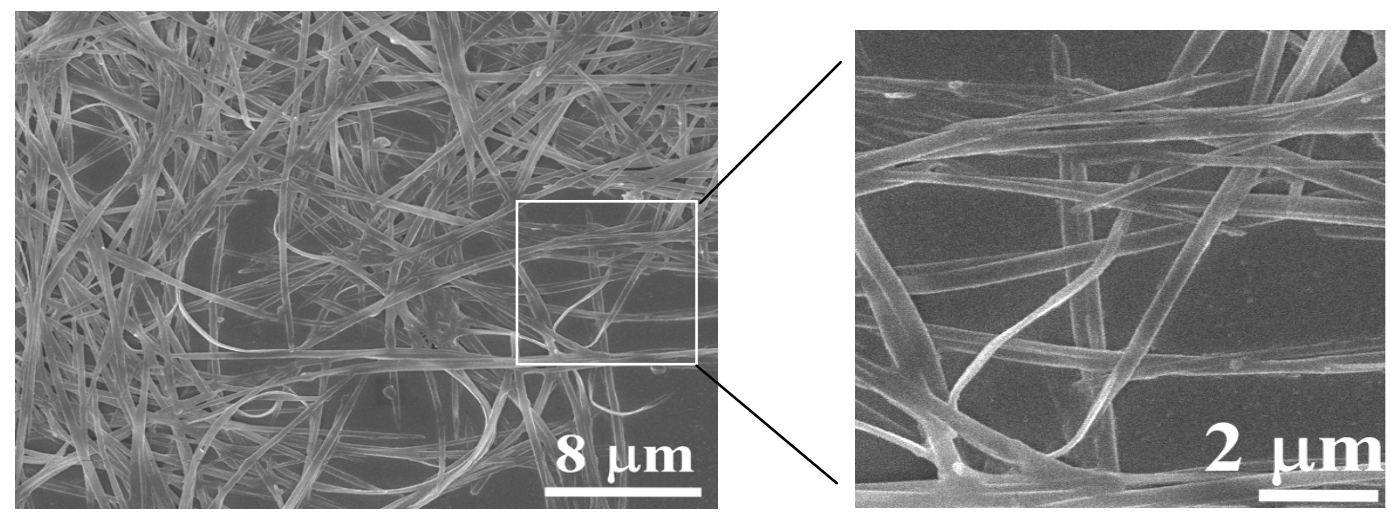

Figure S6. SEM image showing twisted

\section{TEM Measurement}

TEM measurement was performed with a Hitachi 7100 (operated at $75 \mathrm{kV}$ ) and JEOL 2010F (a field emission TEM, operated at $200 \mathrm{kV}$ ). The sample was prepared by casting a methanol solution of PE-PTCDI nanobelts ( $6 \mu \mathrm{M}$ or $25 \mu \mathrm{M}$, aged 1 hour) onto $\mathrm{SiO}_{2}$ film, followed by drying in air. The dried sample was then annealed overnight in an oven at $45{ }^{\circ} \mathrm{C}$. For electron diffraction measurement, the sample was prepared on holey carbon film, which gives much less background scattering than $\mathrm{SiO}_{2}$ film. Diffraction patterns were recorded under controlled electron dose with low illumination intensity to reduce radiation damage of the sample. Figure S7A shows a straight nanobelt lying on the $\mathrm{SiO}_{2}$ surface; Figure $\mathrm{S} 7 \mathrm{~B}$ shows a pile of nanobelts on the same surface.

Electron diffraction of the nanobelts showed typical crystalline patterns with sharp diffraction spots. The inset of Fig. $2 \mathrm{C}$ shows an example pattern recorded from a single nanobelt. This 
particular nanobelt is twined. Two distinct reciprocal lattice vectors can be obtained from this pattern, giving two d-spacings, $\mathrm{d} 1=0.865 \mathrm{~nm}$ and $\mathrm{d} 2=0.466 \mathrm{~nm}$. The $\mathrm{d} 1$ is perpendicular to the belt direction. These spacings are consistent with the X-ray diffraction measurement of bulk crystals of the similar PTCDI molecules, such as the one with ethoxypropyl side chains ( $a=16.665 \AA$, $b=$ $8.657 \AA, \mathrm{c}=4.747 \AA)^{11}$ The molecular stacking distance calculated from these crystalline parameters is around $3.4 \AA{ }^{12}$ which is typical for strong $\pi-\pi$ stacking (interaction).

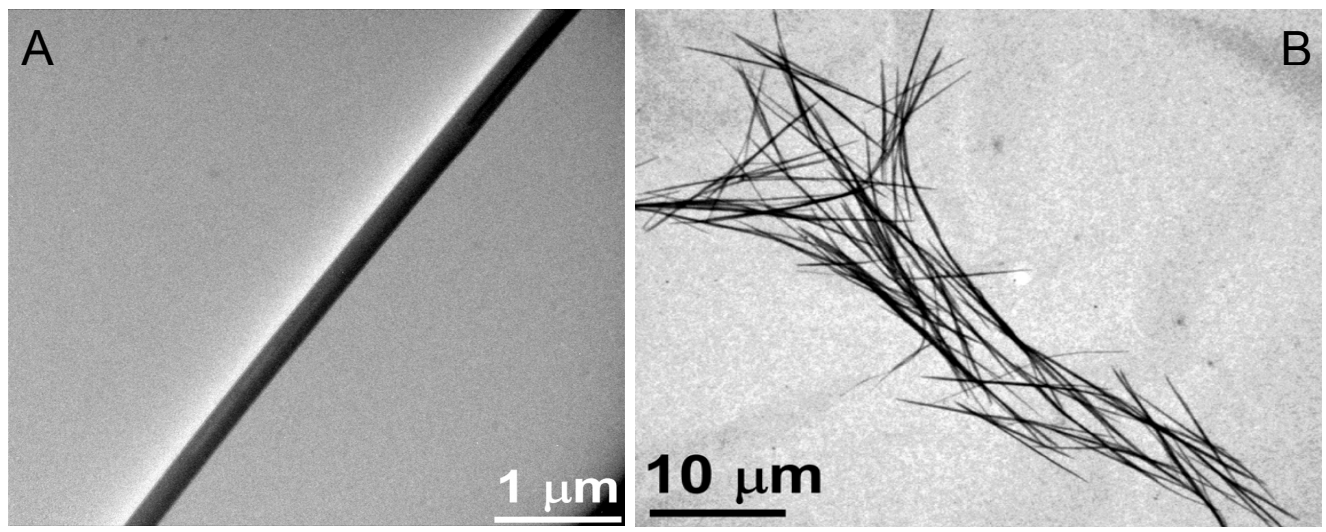

Figure S7. TEM image showing a single nanobelt (A) and a pile of nanobelts (B).

\section{AFM Measurement}

AFM measurement was carried out in tapping mode on a TopoMetrix Explorer using antimony doped silicon tip. The largest scanning area is $50 \times 50 \mu \mathrm{m}$, and the highest z-resolution is about 0.2 $\mathrm{nm}$. The sample was prepared by casting the methanol solution of nanobelts $(6 \mu \mathrm{M}$, aged 1 hour) on mica surface, followed by annealing at $60{ }^{\circ} \mathrm{C}$ for 16 hours. Figure S8 shows a 3D image of a part of one nanobelt shown in Figure 2D in the Communication. It clearly reveals the belt like motif and quite flat surface, which is consistent with the zoom-in TEM imaging (see Figure $2 \mathrm{C}$ in the Communication)

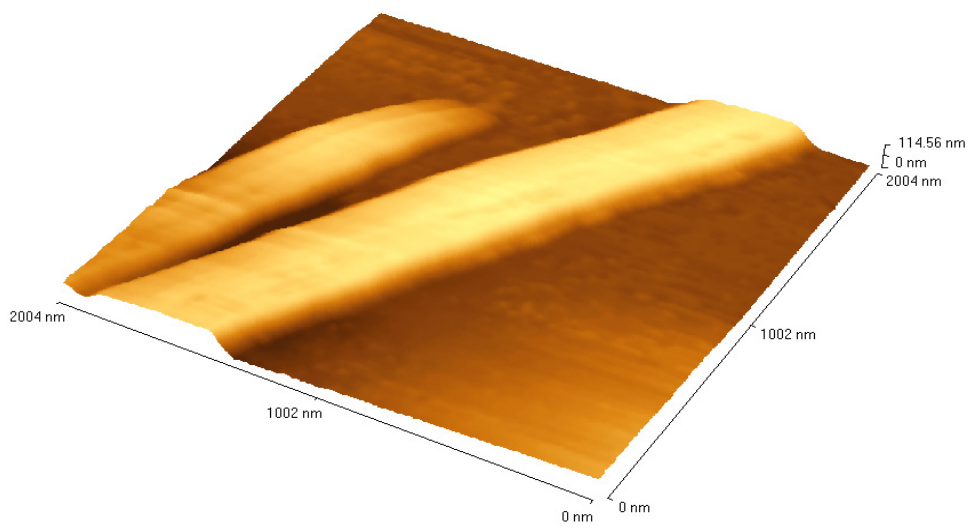

Figure S8. 3D AFM image showing the belt like motif of the self-assembled nanostructure.

Figure S9 depicts two AFM images of the ND-PTCDI nanocrystals formed in 35/65 water/methanol binary solvents. Due to the steric hindrance of the swallow-tail like side chains, no one-dimensional nanostructure was found for ND-PTCDI molecules. Most of the self-assembled crystals were in approximately spherical shape. In the early stage of self-assembly (within one 
hour), the nanospheres were quite small, in the range of 5 to $10 \mathrm{~nm}$ (Figure S9A). Upon prolonged aging in the solution for a week, the particle size increased gradually to about $100 \mathrm{~nm}$ probably through the Oswald ripening, and at the same time the particle shape became more uniform in spherical shape (Figure S9B). The highly symmetric spherical assembly could be the lowest energy state for the molecular packing of ND-PTCDI molecules.
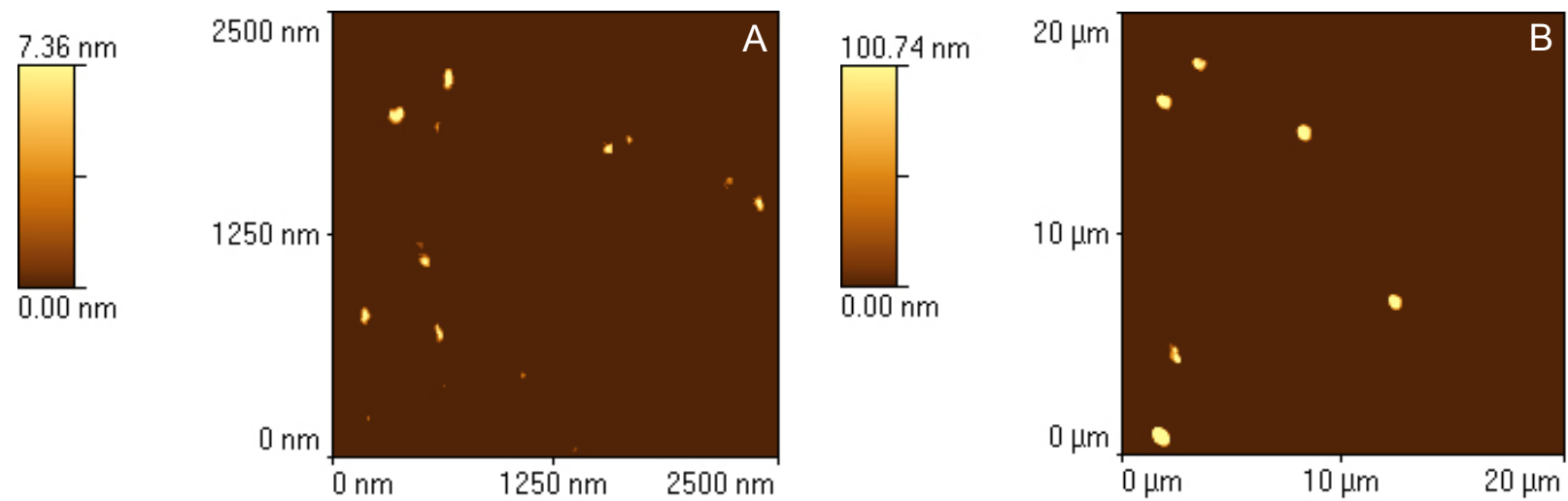

Figure S9. AFM images of the spherical nanocrystals of ND-PTCDI cast on mica surface: (A) the small nanocrystals formed in the first one hour of self-assembly; (B) the ripened nanocrystals aged in the solution for a week.

\section{Polarized Microscopy Imaging}

The polarized optical microscopy imaging was performed with a home built system based on a near-field scanning optical microscope (NSOM) (Aurora III, Veeco). One polarizer was put after the focused white light source which shines the sample perpendicularly from above. The transmitted light was collected with an oil immersion objective (100×, NA 1.25, Olympus) and then imaged with a CCD camera (36× zoom, Nikon). Another polarizer (orthogonal to the other one) was put before the camera for obtaining the cross polarized (dark field) image. To change the relative orientation between the nanobelt and the polarizer, the two polarizers (maintained orthogonal to each other) were rotated parallely the same number of degrees. The bright field image was obtained simply by adjusting the two polarizers in parallel.
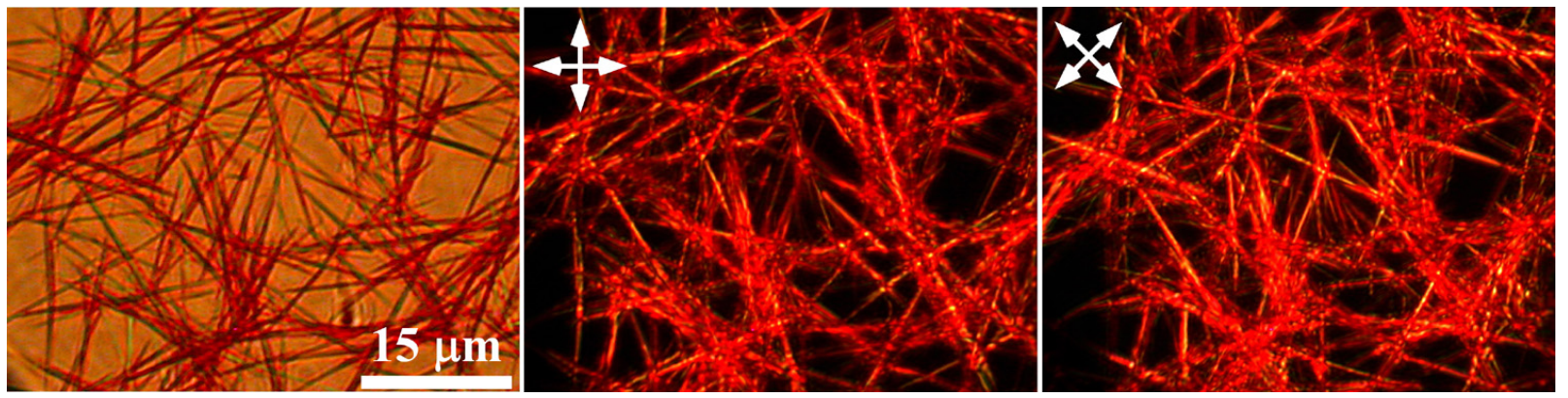

Figure S10. Polarized optical microscopy images of nanobelts. (left) bright filed; (central) dark field; (right) dark field with $45^{\circ}$ rotation of sample.

The sample was prepared by casting the methanol solution of nanobelts $(6 \mu \mathrm{M})$ on a glass cover slip cleaned with piranha reagent (30:70 $\left.\mathrm{H}_{2} \mathrm{O}_{2}(35 \%): \mathrm{H}_{2} \mathrm{SO}_{4}\right)$. Caution: Piranha solution is an extremely strong oxidizing reagent. The dried sample was put on the scanning stage of the 
microscope. The motor controlled stage allows for large area sample translation $(3 \times 3 \mathrm{~mm})$ in micrometer steps. This affords easy selection and positioning of nanobelts for imaging.

Figure S10 shows the bright field and dark field images of a pile of nanobelts. It can be seen that some of the nanobelts showed very low brightness in the dark field (central image), which implies a position of extinction (i.e. optical axis of nanobelt oriented parallel to the direction of the polarizer). With rotating the relative orientation between sample and polarizers by $45^{\circ}$, these dark nanobelts turned to be bright, and on the other hand, the originally bright nanobelts now turned to be dimmer (right image). This observation is consistent with the results obtained by consecutive rotation of the sample in steps of $45^{\circ}$ (Figure 3 in the Communication).

(1) Langhals, H. Heterocycles 1995, 40, 477-500.

(2) Ahrens, M. J.; Sinks, L. E.; Rybtchinski, B.; Liu, W.; Jones, B. A.; Giaimo, J. M.; Gusev, A. V.; Goshe, A. J.; Tiede, D. M.; Wasielewski, M. R. J. Am. Chem. Soc. 2004, 126, 8284-8294.

(3) Van Herrikhuyzen, J.; Syamakumari, A.; Schenning, A. P. H. J.; Meijer, E. W. J. Am. Chem. Soc. 2004, 126, 10021-10027.

(4) Wurthner, F. Chem. Commun. 2004, 1564-1579.

(5) Nguyen, T.-Q.; Martel, R.; Avouris, P.; Bushey, M. L.; Brus, L.; Nuckolls, C. J. Am. Chem. Soc. 2004, 126, 5234-5242.

(6) Zhao, D.; Moore, J. S. Chem. Commun. 2003, 807-818.

(7) Hill, J. P.; Jin, W.; Kosaka, A.; Fukushima, T.; Ichihara, H.; Shimomura, T.; Ito, K.; Hashizume, T.; Ishii, N.; Aida, T. Science 2004, 304, 1481-1483.

(8) Kastler, M.; Pisula, W.; Wasserfallen, D.; Pakula, T.; Mullen, K. J. Am. Chem. Soc. 2005, 127, 4286-4296.

(9) Cormier, R. A.; Gregg, B. A. Chem. Mater. 1998, 10, 1309-1319.

(10) Struijk, C. W.; Sieval, A. B.; Dakhorst, J. E. J.; van Dijk, M.; Kimkes, P.; Koehorst, R. B. M.; Donker, H.; Schaafsma, T. J.; Picken, S. J.; van de Craats, A. M.; Warman, J. M.; Zuihof, H.;

Sudholter, E. J. R. J. Am. Chem. Soc. 2000, 122, 11057-11066.

(11) Hadicke, E. H.; Graser, F. Acta Cryst. 1986, C42, 189-195.

(12) Klebe, G.; Graser, F.; Hadicke, E.; Berndt, J. Acta Cryst. 1989, B45, 69-77.

\section{Complete references cited in the Communication:}

(4) Lee, H. J.; Jin, Z. X.; Aleshin, A. N.; Lee, J. Y.; Goh, M. J.; Akagi, K.; Kim, Y. S.; Kim, D. W.; Park, Y. W. J. Am. Chem. Soc. 2004, 126, 16722.

(7) Hill, J. P.; Jin, W.; Kosaka, A.; Fukushima, T.; Ichihara, H.; Shimomura, T.; Ito, K.; Hashizume, T.; Ishii, N.; Aida, T. Science 2004, 304, 1481.

(10) Katz, H. E.; Lovinger, A. J.; Kloc, C.; Siegrist, T.; Li, W.; Lin, Y.-Y.; Dodabalapur, A. Nature 2000, 404, 478.

(14) Ahrens, M. J.; Sinks, L. E.; Rybtchinski, B.; Liu, W.; Jones, B. A.; Giaimo, J. M.; Gusev, A. V.; Goshe, A. J.; Tiede, D. M.; Wasielewski, M. R. J. Am. Chem. Soc. 2004, 126, 8284. 